\title{
Neonatal vulnerability to ischemia and reperfusion: Cardioplegic arrest causes greater myocardial apoptosis in neonatal lambs than in mature lambs
}

\author{
Mohsen Karimi, MD \\ Li Xing Wang, MD, $\mathrm{PhD}^{\mathrm{a}}$ \\ James M. Hammel, MD ${ }^{a}$ \\ Christopher E. Mascio, MD ${ }^{a}$ \\ Mohamed Abdulhamid, BS $^{\mathrm{a}}$ \\ Elesa W. Barner, MAT ${ }^{\mathrm{a}}$ \\ Thomas D. Scholz, MD ${ }^{\mathrm{b}}$ \\ Jeffrey L. Segar, MD \\ Wei Gen Li, MD, PhD ${ }^{\mathrm{c}, \mathrm{d}}$ \\ Scott D. Niles, CCPa \\ Christopher A. Caldarone, MD ${ }^{a, e}$
}

From the Division of Cardiothoracic Surgery $^{\mathrm{a}}$ and Departments of Pediatrics, ${ }^{\mathrm{b}}$ Biochemistry, ${ }^{\mathrm{c}}$ and Internal Medicine, ${ }^{\mathrm{d}}$ University of Iowa College of Medicine, Iowa City, Iowa, and the Division of Cardiothoracic Surgery, ${ }^{e}$ Hospital for Sick Children, University of Toronto, Toronto, Ontario, Canada.

Supported in part by a Scientist Development Grant from the American Heart Association.

Read at the Eighty-third Annual Meeting of The American Association for Thoracic Surgery, Boston, Mass, May 4-7, 2003.

Received for publication May 9, 2003; revisions requested July 14, 2003; accepted for publication July 31, 2003.

Address for reprints: Christopher A. Caldarone, MD, Division of Cardiovascular Surgery, The Hospital for Sick Children, 555 University Ave, Suite 1525, Toronto, Ontario, Canada, M5G 1X8 (E-mail: christopher-caldarone@ sickkids.ca).

J Thorac Cardiovasc Surg 2004;127:490-7

$0022-5223 / \$ 30.00$

Copyright $\odot 2004$ by The American Association for Thoracic Surgery

doi:10.1016/j.jtcvs.2003.07.052
Objectives: Apoptosis is a mechanism for deletion of injured or obsolete cells that is distinct from necrosis and mediated by mitochondrial release of cytochrome $c$ caspase activation. Because myocardial apoptosis is a part of normal fetal and postnatal maturation, we hypothesize that neonatal myocardium is more vulnerable to undergo myocardial apoptosis than mature myocardium after cardioplegic arrest.

Methods: Newborn and mature lambs $(\mathrm{n}=5$ in each group) underwent cardiopulmonary bypass, antegrade crystalloid hyperkalemic cardioplegic arrest for 60 minutes, and a 6-hour recovery period. Myocardium was examined by using terminal deoxynucleotidyl transferase-mediated deoxyuridine triphosphatedigoxigenin nick end labeling (TUNEL), Western blotting, in vitro kinase assays, and fluorometric assays of the activity of caspases 3, 8, and 9. Myocardium from nonoperated control subjects $(\mathrm{n}=5$ in each age group) was also obtained.

Results: More TUNEL-positive nuclei were present in the newborn postcardioplegic myocardium $(P=.04)$. Caspase 3,8 , and 9 activities were 1.6-fold, 1.5 -fold, and 1.4-fold greater in the newborn postcardioplegic myocardium $(P=$ $.04, P=.01$, and $P=.01$, respectively). The $\mathrm{Bax} / \mathrm{Bcl}-2$ ratio was higher in the newborn postcardioplegic myocardium $(P=.04)$. Apoptosis signal-regulating kinase 1 activity and cleaved caspase 3 levels were higher in the newborn postcardioplegic myocardium $(P=.02$ and $P=.009)$. Mitochondrial release of cytochrome $c$ was greater in the newborn postcardioplegic myocardium $(P=$ $.009)$.

Conclusions: The increased Bax/Bcl-2 ratio in the newborn myocardium suggests a proapoptotic state that is manifested by greater TUNEL staining, cytochrome $c$ release, and cleavage of caspase 3. Increased apoptosis signal-regulating kinase 1 activity suggests greater oxidative stress, immature mechanisms to ameliorate oxidative stress, or both in the neonatal myocardium. Mitochondrial release of cytochrome $c$ suggests that apoptosis-related mitochondrial dysfunction might contribute to early postoperative myocardial dysfunction in the neonate. 


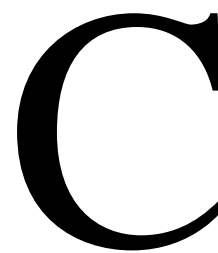

ardiac surgery in the neonate is frequently followed by early postoperative myocardial dysfunction. This myocardial dysfunction typically peaks at 6 to 12 hours after the operation and improves thereafter. ${ }^{1,2}$ Although postoperative dysfunction in general has been ascribed to myocardial stunning, the relationship between classical models of stunning and the "midnight sag" has not been clearly defined.

In addition to myocardial stunning, the neonatal heart might be uniquely vulnerable to initiation of apoptosis in the early postoperative period for the following reasons. First, neonatal hearts activate apoptosis pathways as a normal part of postnatal myocardial remodeling. ${ }^{3}$ Second, cardiopulmonary bypass causes a systemic increase of proapoptotic factors (eg, tumor necrosis factor $\alpha$ ). ${ }^{4}$ Finally, the ischemiareperfusion injury associated with cardioplegic arrest might initiate apoptosis. ${ }^{5}$ In addition, the time course of the mitochondrial dysfunction associated with apoptosis coincides with the time course of the midnight sag. ${ }^{6}$ Consequently, we hypothesize that activation of apoptosis-related mechanisms is an important consequence of cardiac surgery in the neonate. Because activation of apoptosis is intimately associated with release of cytochrome $c$ from the mitochondria and diminished mitochondrial energy production, ${ }^{7}$ apoptosis-related processes might contribute to the clinical observation of decreased myocardial function in the early postoperative period. ${ }^{2}$

In this study we explored age-related differences in the myocardial response to cardiopulmonary bypass and cardioplegic arrest in a clinically relevant sheep model. Specifically, we seek to compare the balance between proapoptotic and antiapoptotic mediators in the neonatal and mature myocardium, as well as to demonstrate the association between the proapoptotic state and the apoptosis-related manifestations of cardioplegic arrest and 6 hours of reperfusion. We hypothesize that the proapoptotic state of the newborn myocardium will lead to greater activation of apoptosis mediators, more extensive apoptosis-related mitochondrial alterations, and a greater proportion of myocytes undergoing completion of apoptosis than mature myocardium.

\section{Materials and Methods}

\section{Surgical Preparation}

Six- to 8-day-old lambs (newborn group, $\mathrm{n}=5$ ) and 6- to 8-weekold lambs (mature group, $\mathrm{n}=5$ ) were anesthetized with intravenous sodium pentothal, intubated orotracheally, and maintained with inhalational general anesthesia for the duration of the experiment. Through a median sternotomy, right atrial and aortic cannulas were placed, and cardiopulmonary bypass was initiated with a blood prime and passive cooling to a core temperature of $28^{\circ} \mathrm{C}$ to $30^{\circ} \mathrm{C}$. The ductus arteriosis was ligated if patent. The aorta was then crossclamped, and cold Plegisol (Abbott's cardioplegic solu- tion: $\mathrm{Na}, 110 \mathrm{mEq} / \mathrm{L} ; \mathrm{Cl}, 160 \mathrm{mEq} / \mathrm{L} ; \mathrm{K}, 16 \mathrm{mEq} / \mathrm{L} ; \mathrm{Ca}, 2.4$ $\mathrm{mEq} / \mathrm{L} ; \mathrm{Mg}, 32 \mathrm{mEq} / \mathrm{L} ; \mathrm{pH} 7.8$ ) was delivered at $4^{\circ} \mathrm{C}$ into an aortic root needle with 50 to $70 \mathrm{~mm} \mathrm{Hg}$ of perfusion pressure to a total initial dose of $20 \mathrm{~mL} / \mathrm{kg}$ body weight. Subsequent cold cardioplegia doses of $15 \mathrm{~mL} / \mathrm{kg}$ were delivered at 20-minute intervals. Between cardioplegia doses, topical cooling was applied, and the aortic root and pulmonary artery were vented. After 60 minutes of arrest, the crossclamp was removed, the animal was rewarmed, and, after a 10- to 20-minute stabilization period, bypass was terminated, heparin was reversed with protamine, and the perfusion cannulas were removed.

General anesthesia was maintained for 6 hours after reperfusion, during which time arterial blood gases, electrolytes, and hematocrit levels were maintained in a physiologic range, and maternal blood or crystalloid was infused as necessary to maintain arterial pressure. Inotropic agents were not administered. After 6 hours, the heart was quickly excised and perfused through the coronary arteries with $240 \mathrm{~mL}$ of ice-cold Dulbecco's phosphatebuffered saline. Full-thickness left ventricular free wall was minced and snap-frozen in liquid nitrogen and then stored at $-80^{\circ} \mathrm{C}$. Nonoperated animals for each age group were anesthetized, and cardiectomy was performed shortly afterward through a median sternotomy. Tissue samples were treated in the same fashion as experimental groups. All animals appropriately received humane care in compliance with the "Guide for the Care and Use of Laboratory Animals" prepared by the Association for Assessment and Accreditation of Laboratory Animal Care (March 1999).

\section{Tissue Samples}

Immediately after death, myocardial tissue samples (approximately $1.0 \times 1.5 \mathrm{~mm}$ each) were sliced, snap-frozen in liquid nitrogen, and stored at $-80^{\circ} \mathrm{C}$ for Western blotting and enzymatic studies. Other tissues were fixed fresh in $10 \%$ formaldehyde overnight and subsequently embedded in paraffin for terminal deoxynucleotidyl transferase-mediated deoxyuridine triphosphatedigoxigenin nick end labeling (TUNEL) study.

\section{Tissue Fractionation for Biochemical Analysis}

Approximately $200 \mathrm{mg}$ of frozen left ventricular myocardial samples were mechanically homogenized with Dounce homogenizer in ice-cold isotonic buffer $(250 \mathrm{mmol} / \mathrm{L}$ sucrose, $20 \mathrm{mmol} / \mathrm{L} \mathrm{N}-2$ hydroxyethylpiperazine-N-2-ethanesulfonic acid, $10 \mathrm{mmol} / \mathrm{L} \mathrm{KCl}$, $1.5 \mathrm{mmol} / \mathrm{L} \mathrm{MgCl} 2,1 \mathrm{mmol} / \mathrm{L}$ ethylenediamine tetraacetic acid, 1 $\mathrm{mmol} / \mathrm{L}$ ethyleneglycol-bis-[ $\beta$-aminoethylether]-N,N,N',N'-tetraacetic acid, $1 \mathrm{mmol} / \mathrm{L}$ dithiothreitol [DTT], $17.4 \mu \mathrm{g} / \mathrm{mL}$ phenylmethylsulfonyl fluoride [PMSF], $8 \mu \mathrm{g} / \mathrm{mL}$ aprotinin, and $2 \mu \mathrm{g} / \mathrm{mL}$ leupeptin [pH 7.4]). The cells were lysed by using 10 loose and then 10 tight strokes in an ice-cold cylinder Dounce homogenizer. Unlysed cells and nuclei were removed by means of centrifugation at $750 \mathrm{~g}$ for 5 minutes at $4^{\circ} \mathrm{C}$. The supernatant was spun at $10,000 \mathrm{~g}$ for 25 minutes at $4^{\circ} \mathrm{C}$, and the resulting mitochondrial pellets were then resuspended in the above buffer and frozen in multiple samples at $-80^{\circ} \mathrm{C}$. The supernatant was centrifuged at $100,000 \mathrm{~g}$ for 1 hour at $4^{\circ} \mathrm{C}$. The supernatant of this final centrifugation, representing the cytosolic fraction, was divided into aliquots and stored at $-80^{\circ} \mathrm{C}$ for future analysis. 


\section{In Situ TUNEL}

TUNEL was performed with a modification of the technique described by Olivetti and colleagues ${ }^{8}$ by using the ApopTag InSitu kit (Intergen). Briefly, 5- $\mu \mathrm{m}$ cryostat sections were fixed with freshly prepared $10 \%$ formaldehyde at room temperature overnight and then paraffinized. The TUNEL assay was performed in 5- $\mu \mathrm{m}-$ thick deparaffinized sections. Four 5- $\mu \mathrm{m}$ sections were preincubated with equilibration buffer for 5 minutes and subsequently incubated with deoxyribonucleotidyl transferase in the presence of digoxigenin-conjugated deoxyuridine triphosphate for 1 hour at $37^{\circ} \mathrm{C}$. The reaction was terminated by incubating the samples in stopping buffer and then with the fluorescein-labeled antidigoxigenin antibody (yellow-green). Cell nuclei were counterstained with DAPI (Vector Laboratories). Ten random high-power fields representing approximately 4000 cells were counted to enumerate TUNEL-positive cells. Formalin-fixed and paraffin-embedded $5-\mu \mathrm{m}$ sections were stained with mouse monoclonal IgM anti- $\alpha$ sarcomeric actin antibody (Sigma) and Alexa Fluor 594-conjugated goat anti-mouse IgM (Molecular Probes) and then TUNEL stained as above to identify cell type. Adjacent sections were stained with hematoxylin and eosin and examined by means of light microscopy for evidence of necrosis.

\section{Caspase Activity Assay}

A fluorometric caspase activity assay was performed as previously described. ${ }^{9}$ Snap-frozen left ventricular myocardial samples were homogenized in cold detergent buffer $(10 \mathrm{mmol} / \mathrm{L}$ Tris- $\mathrm{HCl}, \mathrm{pH}$ $8.0 ; 1 \%$ Triton $\mathrm{X}-100 ; 0.32 \mathrm{~mol} / \mathrm{L}$ sucrose; and $5 \mathrm{mmol} / \mathrm{L}$ ethylenediamine tetraacetic acid) with proteinase inhibitors $(1 \mathrm{mmol} / \mathrm{L}$ PMSF, $2 \mathrm{mmol} / \mathrm{L}$ DTT, $10 \mu \mathrm{g} / \mathrm{mL}$ leupeptin, and $10 \mu \mathrm{g} / \mathrm{mL}$ aprotinin) and cellular debris pelleted at $13,000 \mathrm{~g}$ for 10 minutes at $4^{\circ} \mathrm{C}$. Supernatant protein concentration was standardized on the basis of chromogenic assay (Bio-Rad). Samples were diluted in assay buffer (10\% sucrose; $100 \mathrm{mmol} / \mathrm{L} \mathrm{N}$-2-hydroxyethylpiperazine-N-2-ethanesulfonic acid, $\mathrm{pH} 7.5 ; 100 \mathrm{mmol} / \mathrm{L} \mathrm{NaCl}$; and $0.1 \%$ CHAPS) with proteinase inhibitors as above. Tetrapeptide substrates of caspase 3 (Ac-DEVD-AFC), caspase 8 (CBZ-IETDAFC), and caspase 9 (Ac-LEHD-AFC; all from Sigma) were added $(50 \mu \mathrm{mol} / \mathrm{L})$, and the fluorescence before and after 1 hour of incubation at $37^{\circ} \mathrm{C}$ were measured with a PerkinElmer LM50B fluorometer $(\mathrm{EG \& G})$ and compared with a curve of known concentrations diluted in each sample.

\section{Citrate Synthase Activity}

Citrate synthase activity was determined by using acetyl coenzyme A solution (12.5 mmol/L, $\mathrm{pH} 5)$ and oxaloacetic acid solution (OAA; $10 \mathrm{mmol} / \mathrm{L}$ ) with 5,5'-dithio-bis(2-nitrobenzoic acid) solution $(1 \mathrm{mmol} / \mathrm{L}$ in $1 \mathrm{~mol} / \mathrm{L}$ Tris- $\mathrm{HCl}, \mathrm{pH} 8.1)$ as a reporting molecule. A quantity of $10 \mu \mathrm{L}$ of cytosolic protein was incubated with $810 \mu \mathrm{L}$ of ddH20, $100 \mu \mathrm{L}$ of $1 \mathrm{mmol} / \mathrm{L} 5,5$ '-dithio-bis(2nitrobenzoic acid), and $30 \mu \mathrm{L}$ of acetyl coenzyme A. The rate before the addition of OAA was measured for 2 minutes by using a kinetics program in conjunction with a Beckman Spectrophotometer, with an absorption wavelength set at $405 \mathrm{~nm}$. After 2 minutes, $50 \mu \mathrm{L}$ of OAA was added, and the activity was then measured as a difference in rates before and after OAA addition. Citrate synthase activity was calculated by dividing this number by the protein concentration.

\section{Western Blotting for Cytochrome $c$, Bax, Bcl-2, Bcl-x ${ }_{L}$, and Caspase 3}

Homogenized samples standardized for protein content were subjected to sodium dodecylsulfate-polyacrylamide gel electrophoresis on $15 \%$ linear gradient gels and subsequently transferred to nitrocellulose membranes. The membrane was probed for cytochrome $c$ with monoclonal anti-mouse cytochrome $c$ antibody (BD Biosciences Pharmingen). The membrane was probed for Bax, Bcl-2, and Bcl- $\mathrm{x}_{\mathrm{L}}$ with anti-mouse IgG monoclonal antibodies (Santa Cruz Biotechnology). For quantitation of total and cleaved caspase 3, the membrane was probed with rabbit anti-human caspase 3 antibody (New England Biolabs Inc). Secondary antibodies were coupled to horseradish peroxidase (anti-mouse IgGhorseradish peroxidase, Upstate Biotechnology; anti-rabbit IgGhorseradish peroxidase, Santa Cruz Biotechnology). The resulting autoradiographs were scanned and quantified with a densitometer (National Institutes of Health image software).

\section{Measurement of Apoptosis-regulating Kinase 1 Activity}

Left ventricular myocardium was homogenized on ice in cell lysis buffer $(50 \mathrm{mmol} / \mathrm{L}$ Tris- $\mathrm{HCl}$ [pH 7.5], $150 \mathrm{mmol} / \mathrm{L} \mathrm{NaCl}, 10$ $\mathrm{mmol} / \mathrm{L}$ ethylenediamine-N',N',N',N'-tetraacetic acid, $0.2 \mathrm{mmol} / \mathrm{L}$ DTT, $200 \mu \mathrm{g} / \mathrm{mL}$ leupeptin, $20 \mu \mathrm{g} / \mathrm{mL}$ aprotinin, and $1 \mathrm{mmol} / \mathrm{L}$ PMSF). After incubation at $4{ }^{\circ} \mathrm{C}$ for 30 minutes, the homogenates were sonicated on ice for 1 minute and then centrifuged at 15,000 $\mathrm{rpm}$ at $4^{\circ} \mathrm{C}$ for 30 minutes. After centrifugation, the supernatants were stored at $-80^{\circ} \mathrm{C}$ until later use. Triton $\mathrm{X}-100$ (1\% final concentration) was added to protein extracts $(50 \mu \mathrm{g})$, followed by incubation with antibody against apoptosis-regulating kinase 1 (ASK-1; pc rabbit, Santa Cruz Biotechnology). The immune complex was precipitated with protein A agarose at $4^{\circ} \mathrm{C}$ for 1 hour. The precipitated ASK-1 was washed 3 times with $20 \mathrm{mmol} / \mathrm{L}$ Tris- $\mathrm{HCl}$ (pH 7.5) buffer containing $150 \mathrm{mmol} / \mathrm{L} \mathrm{NaCl}, 5 \mathrm{mmol} / \mathrm{L}$ bis$\mathrm{N}, \mathrm{N}, \mathrm{N}^{\prime}, \mathrm{N}^{\prime}$-tetraacetic acid, $2 \mathrm{mmol} / \mathrm{L} \mathrm{DTT}$, and $1 \mathrm{mmol} / \mathrm{L} \mathrm{PMSF}$ and once with a reaction buffer of $20 \mathrm{mmol} / \mathrm{L}$ Tris- $\mathrm{HCl}(\mathrm{pH} \mathrm{7.5)}$ and $20 \mathrm{mmol} / \mathrm{L} \mathrm{MgCl}_{2}$. The reaction was carried out in the reaction buffer in the presence of $0.5 \mu \mathrm{Ci}$ of phosphorous 32-labeled adenosine triphosphate (ATP; PerkinElmer Life Sciences) and 1 $\mathrm{mmol} / \mathrm{L}$ ATP for 30 minutes at $30^{\circ} \mathrm{C}$ by using myelin basic protein (40 $\mu \mathrm{g} / \mathrm{mL}$, Sigma) as exogenous substrate. The phosphorylated proteins were separated by using $15 \%$ sodium dodecylsulfatepolyacrylamide gel electrophoresis and visualized with autoradiography. The resulting images were scanned and analyzed with National Institutes of Health image software.

\section{Statistical Analysis}

Data are presented as means \pm SEM, and comparisons between the 2 main groups and subgroups were made by using analysis of variance, followed by the Student $t$ test.

\section{Results \\ TUNEL}

The number of TUNEL-positive nuclei in left ventricular sections after cardioplegia is depicted in Figure 1. There were $3.4 \pm 1.6$ positive nuclei per high-power field in the newborn cardioplegia group (representing approximately 


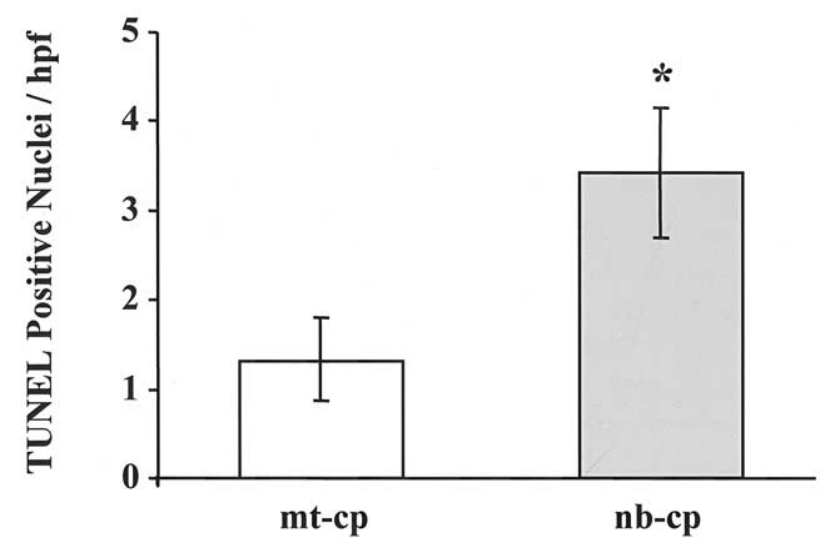

Figure 1. The number of TUNEL-positive nuclei per high-power field in left ventricular myocardium after cardioplegia in newborn ( $n b-c p)$ and mature (mt-cp) lambs. Cryostat sections were stained by using TUNEL and counterstained with DAPI. Fluorescent nuclei in $\mathbf{1 0}$ random high-power fields per section were manually counted. TUNEL positivity in newborns was significantly greater than in mature cardioplegic lambs. All results are shown as means \pm SEM ( $n=5$ for each sample). *Significant difference at a $P$ value of less than .05 .

$0.82 \%$ of cells) compared with $1.3 \pm 1.0$ per high-power field in mature cardioplegic lambs $(P=.04)$. There were no TUNEL-positive nuclei in the nonoperated groups (results not shown). Labeling with TUNEL, DAPI, and actin immunofluorescence showed that nearly all TUNEL-positive nuclei appeared in actin-containing cells, indicating cardiomyocyte apoptosis. Adjacent sections stained with hematoxylin and eosin showed no evidence of necrosis (results not shown).

\section{Caspase Activity}

Caspase 3-, 8-, and 9-like activities are shown in Figure 2. Six hours after cardioplegia, caspase 3, 8, and 9 activities were 1.6-fold, 1.5-fold, and 1.4-fold greater in neonates $(P$ $=0.04, P=.01$, and $P=.01$, respectively) than in mature cardioplegic lambs.

\section{Caspase 3 Western Blotting}

Western blot analysis showed a significant increase in the concentration of cleaved caspase 3 (the active form) in the neonatal cardioplegic group compared with that in the mature cardioplegic group $(26.6 \pm 4.3 \mathrm{AU}$ vs $6.05 \pm 3.2 \mathrm{AU}$, $P=.009)$. There were no significant differences in total caspase 3 (inactive form) levels between the 2 groups (124.3 $\pm 10.4 \mathrm{AU}$ vs $125.8 \pm 6.8 \mathrm{AU}, P=.91$, Figure 3 ).

\section{Cytosolic Cytochrome $\boldsymbol{c}$ Western Blotting}

After subcellular fractionation, Western immunoblot analysis for cytosolic cytochrome $c$ revealed higher concentrations of cytosolic cytochrome $c$ in the newborn cardioplegic

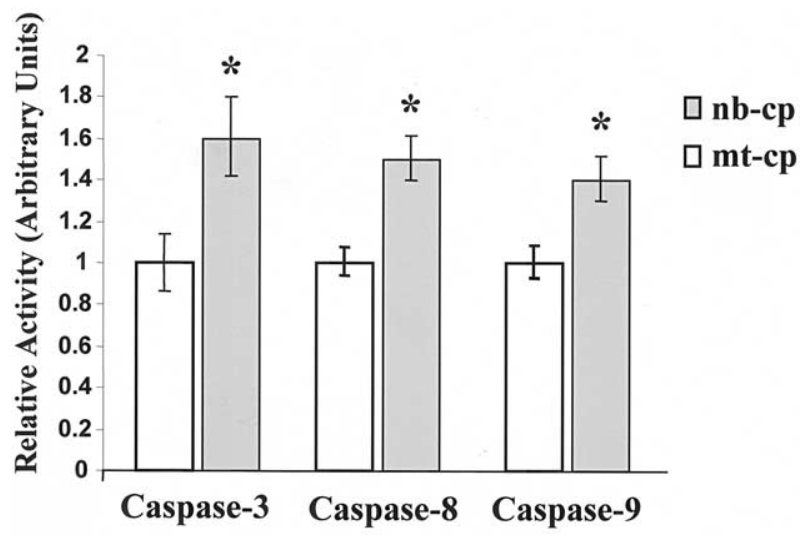

Figure 2. Hydrolytic activity for specific tetrapeptide substrates of caspases 3, 8, and 9 in total cellular protein from left ventricular myocardium of newborn (nb-cp) and mature (mt-cp) cardioplegic lambs. Activity is expressed graphically as the ratio of activity in newborn to mature protein extracts for each caspase. All results are shown as means \pm SEM ( $n=5$ for each sample). *Significant difference at a $\boldsymbol{P}$ value of less than .05 .

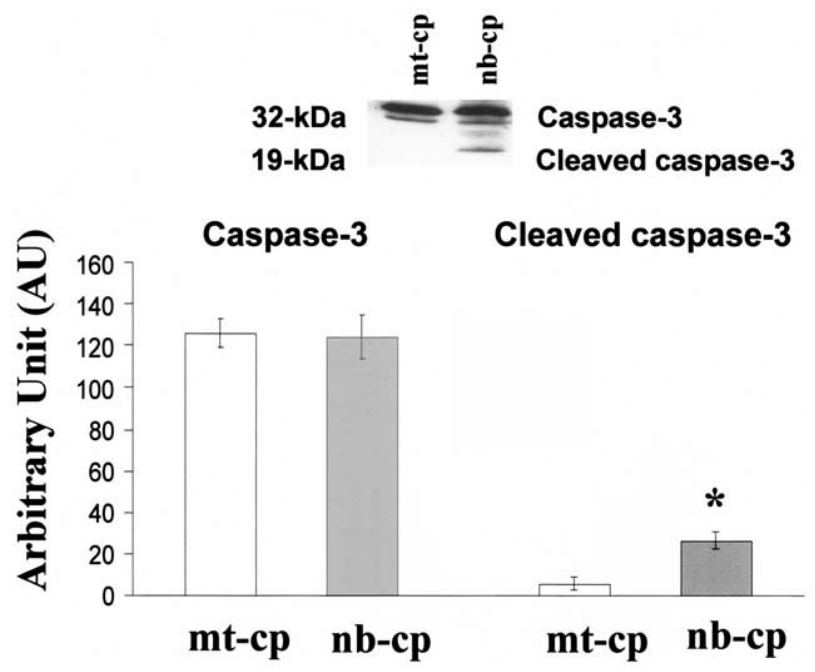

Figure 3. Western blot densitometric measurements of total caspase 3 and cleaved caspase 3 in the mature cardioplegic (mt-cp) group versus the newborn cardioplegic (nb-cp) group. Representative Western immunoblots for each group are shown above each bar graph. All results are shown as means \pm SEM ( $n$ $=\mathbf{5}$ for each sample). *Significant difference at a $\boldsymbol{P}$ value of less than 05 .

group compared with in the mature cardioplegic group. These data were normalized to citrate synthase activity, which was used to correct for artifactual disruption of the mitochondria during the tissue preparation process. After normalization, the cytosolic cytochrome $c$ concentration was greater in the newborn cardioplegic group than in the mature cardioplegic group $\left(146.2 \pm 24 \mathrm{AU} \cdot \mathrm{U}^{-1} \cdot \mathrm{mg}^{-1} \mathrm{vs}\right.$ 


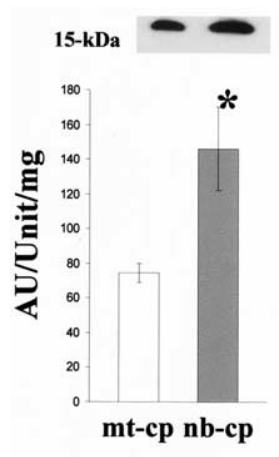

A

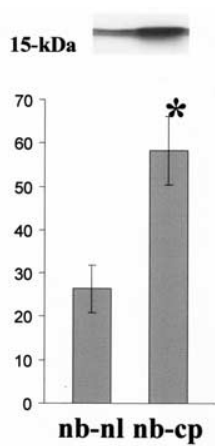

B
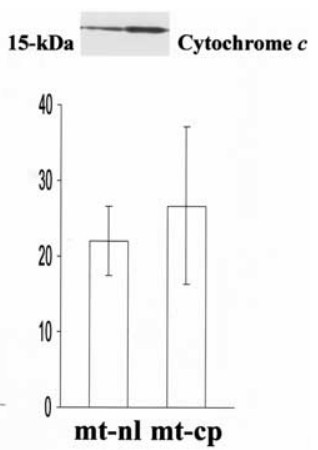

C
Figure 4. Western blot densitometric measurements of cytosolic cytochrome $c$ normalized to citrate synthase for mature (mt) and newborn (nb) myocardium harvested from nonoperated (nl) and postcardioplegia (cp) hearts. Representative Western immunoblots for each comparison are shown above the bar graph. All results are shown as means \pm SEM ( $n=5$ for each comparison). *Significant difference at a $\boldsymbol{P}$ value of less than .05 .

$74.5 \pm 5.6 \mathrm{AU} \cdot \mathrm{U}^{-1} \cdot \mathrm{mg}^{-1}, P=.009$; Figure 4, A). Our measurements displayed some citrate synthase activity in all cytosolic preparations ranging between 0.46 and $3.97 \mathrm{U} / \mathrm{mg}$ protein. We also used cytochrome $c$ oxidase subunit IV (Cox-4) as another indicator of mitochondrial membrane disruption during tissue homogenization. Western blotting of the cytosolic fraction failed to show the presence of Cox-4 for all groups, indicating the relative purity of the tissue fractions (results not shown). Greater concentration of cytosolic cytochrome $c$ normalized to citrate synthase activity was found in the newborn cardioplegic group when compared with that seen in newborn nonoperated animals $\left(58.3 \pm 7.9 \mathrm{AU} \cdot \mathrm{U}^{-1} \cdot \mathrm{mg}^{-1}\right.$ vs $26.4 \pm 5.5 \mathrm{AU} \cdot \mathrm{U}^{-1}$. $\mathrm{mg}^{-1}, P=.01$; Figure $4, B$ ). In contrast, there were no significant differences in the cytosolic cytochrome $c$ release in the mature cardioplegic group when compared with that in the mature nonoperated animals $\left(26.7 \pm 10.4 \mathrm{AU} \cdot \mathrm{U}^{-1}\right.$ $\cdot \mathrm{mg}^{-1}$ vs $22.04 \pm 4.6 \mathrm{AU} \cdot \mathrm{U}^{-1} \cdot \mathrm{mg}^{-1}, P=.68$; Figure $4, C)$.

\section{Bax and Bcl-2 Western Blotting}

The densitometric data of the Western blotting for Bax and $\mathrm{Bcl}-2$ revealed a higher concentration of Bax protein in the newborn cardioplegic group in comparison with that found in the mature cardioplegic group (108 \pm 10.3 vs $78 \pm 6.8$ AU, $P=.04)$. There were no statistically significant differences in the amount of Bcl-2 for either group (88.9 $\pm 6.5 \mathrm{vs}$ $89 \pm 3.9 \mathrm{AU}, P=.98$ ). Therefore the Bax/Bcl-2 ratio, a measure of proapoptotic state, was greater in the newborn heart compared with that in the mature heart after cardioplegic arrest $(1.22 \pm 0.1$ vs $0.88 \pm 0.09, P=.04$; Figure $5, A)$. Although higher in the neonatal hearts, the $\mathrm{Bax} / \mathrm{Bcl}-2$ ratio

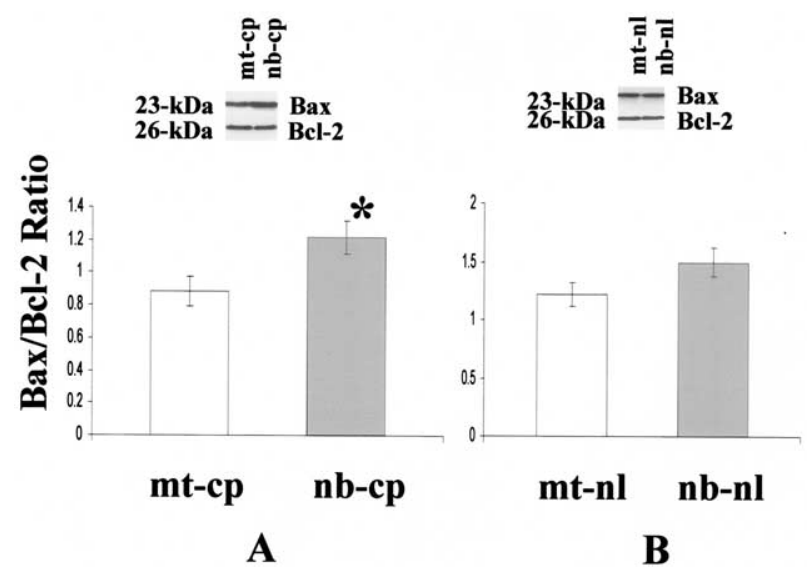

Figure 5. Western blot densitometric measurements of $\mathrm{Bax} / \mathrm{Bcl}-2$ ratio for the mature cardioplegic (mt-cp) versus newborn cardioplegic (nb-cp) groups (A) and mature nonoperated (mt-nl) versus newborn nonoperated (nb-nl) groups (B). Representative Western immunoblots for each group are shown above each bar graph. All results are shown as means \pm SEM ( $n=5$ for each sample). Significant difference at a $\boldsymbol{P}$ value of less than .05 .

was not different when comparing nonoperated groups $(1.50 \pm 0.13$ vs $1.22 \pm 0.01, P=.12$; Figure $5, B)$.

\section{Bcl- $x_{L}$ Western Blotting}

The densitometric data of the Western blot for $\mathrm{Bcl}-\mathrm{x}_{\mathrm{L}}$ revealed a higher concentration of the protein in the newborn cardioplegic group in comparison with that in the mature cardioplegic group $(94.7 \pm 6.3 \mathrm{AU}$ vs $48.6 \pm 5.1 \mathrm{AU}, P=$ .0006; Figure 6, $A$ ). There were no statistically significant differences in the amount of $\mathrm{Bcl}-\mathrm{x}_{\mathrm{L}}$ for nonoperated newborn and mature groups $(72.5 \pm 6.4 \mathrm{AU}$ vs $57.1 \pm 6.4 \mathrm{AU}$, $P=.08$; Figure $6, B)$.

\section{Activation of ASK-1}

ASK-1 activity, a measure of oxidative stress, was greater in the newborn cardioplegic group compared with in the mature cardioplegic group, as measured with an in vitro kinase assay $(115.1 \pm 5.3 \mathrm{AU}$ vs $90.4 \pm 6.2 \mathrm{AU}, P=.02$, Figure 7).

\section{Discussion}

Morphologic, histochemical, and biochemical evidence for the early stages of myocyte apoptosis arising during the course of cardiac surgery have been reported in myocardial samples after cardioplegic arrest and reperfusion in the human heart. ${ }^{10}$ Our previous study showed that myocyte apoptosis was detectable in the left ventricle of the neonatal lamb heart after cardioplegic arrest and reperfusion demonstrated on the basis of TUNEL positivity and DNA laddering. ${ }^{11}$ In the present study results from TUNEL suggest a greater degree of myocardial apoptosis after cardioplegic 


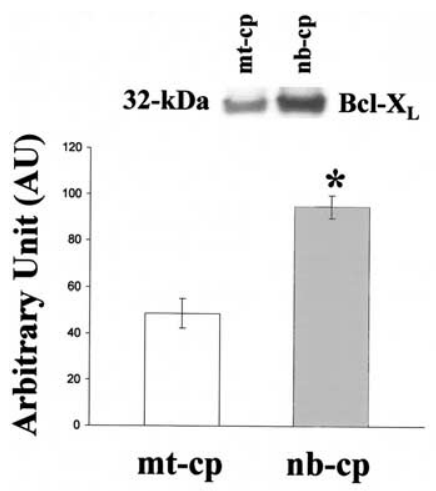

A

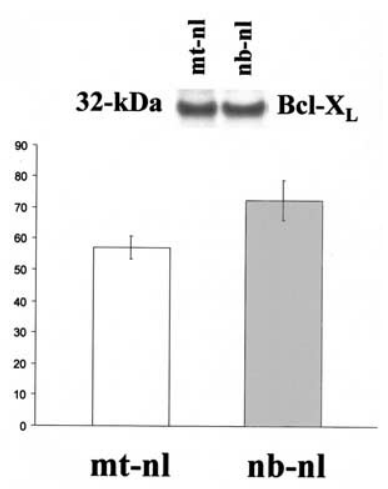

B
Figure 6. Western Blot densitometric measurements of $B c l-x_{L}$ for newborn cardioplegic ( $n b$-cp) versus mature cardioplegic (mt-cp) groups $(A)$ and newborn nonoperated (nb-nl) versus mature nonoperated (mt-nl) groups (B). Representative Western immunoblots for each group are shown above each bar graph. All results are shown as means \pm SEM ( $\mathrm{n}=\mathbf{5}$ for each sample). *Significant difference at a $\boldsymbol{P}$ value of less than .05 .

arrest in the neonatal lamb compared with in more mature lambs.

Caspase 8 is an upstream caspase that transduces proapoptotic signals from the cell-surface death receptor complex in response primarily to inflammatory triggers, such as tumor necrosis factor $\alpha$, which has been shown to be increased after cardiopulmonary bypass. ${ }^{12}$ Caspase 9 , also an upstream caspase, is activated after mitochondrial release of cytochrome $c$ in response to oxidative stress after ischemiareperfusion injury. Caspase 3 , an effector caspase, can be activated through both the above pathways, resulting in the activation of a variety of enzymes and feedback loops and subsequent completion of cell death. In the present study we found that the activities of all 3 caspases were higher in the newborn myocardium than in the mature myocardium after cardioplegic arrest and reperfusion. The difference in caspase activity between newborns and mature lambs after the same surgical procedure therefore might reflect either a difference in activation after cardioplegic arrest or a more proapoptotic status of the newborn myocardium.

The release of cytochrome $c$ from mitochondria is central to all forms of apoptosis, resulting in the downstream activation of effector caspases (ie, caspase 3). ${ }^{13}$ Cytochrome $c$ release is known to be regulated by Bcl-2 family proteins. ${ }^{14}$ Antiapoptotic members of this family, including Bcl-2 and $\mathrm{Bcl}-\mathrm{x}_{\mathrm{L}}$, bind to the mitochondrial outer membrane and inhibit the release of cytochrome $c$ from the mitochondria. In contrast, proapoptotic members of the Bcl-2 family, such as Bax, promote the release of cytochrome $\mathrm{c}$ and other mitochondrial intermembrane proteins. ${ }^{15}$ The release of cytochrome $c$ into the cytoplasm has been widely reported in

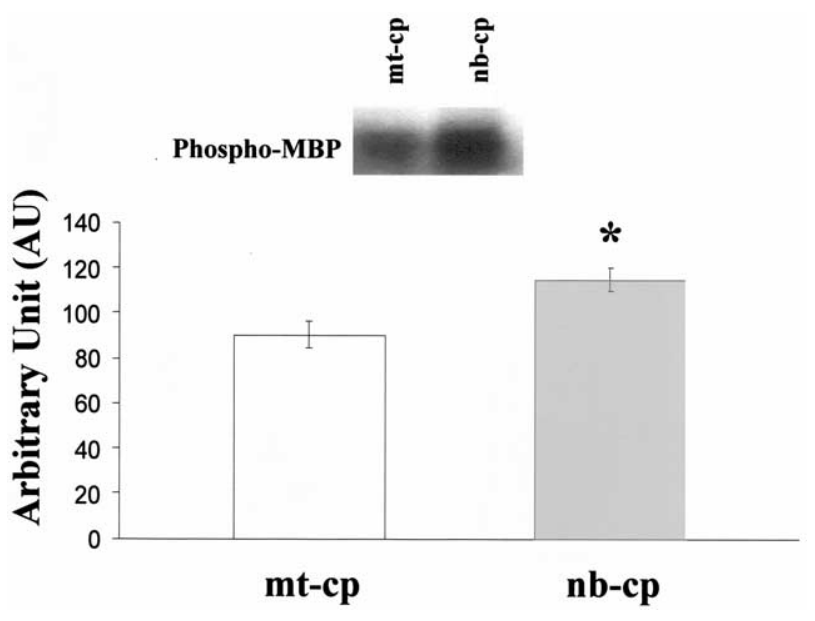

Figure 7. In situ kinase activity for ASK-1 in the mature cardioplegic (mt-cp) group versus the newborn cardioplegic (nb-cp) group. Representative Western immunoblots for each group are shown above the bar graph. All results are shown as means \pm SEM ( $n$ $=\mathbf{5}$ for each sample). * Significant difference at a $\boldsymbol{P}$ value of less than .05 .

multiple studies as an irrevocable commitment to cell death. ${ }^{16-19}$ In contrast, some recent studies have demonstrated that the release of cytochrome $c$ does not invariably lead to apoptotic cell death. ${ }^{20}$

The greater $\mathrm{Bax} / \mathrm{Bcl}-2$ ratio in the neonatal cardioplegic group suggests that the neonatal hearts are in a more proapoptotic state. The lack of change in the $\mathrm{Bax} / \mathrm{Bcl}-2$ ratio when comparing the nonoperated and postoperative states, however, suggests that expression of Bax at the protein level is not upregulated within the early postoperative period, which is consistent with our previous report that Bax expression at the mRNA level was not upregulated in response to cardioplegic arrest in the neonate. ${ }^{12}$ Nevertheless, these data do not exclude the possibility of Bax participation in the mediation of cytochrome $c$ release in this model through translocation from the cytosol to the mitochondrial membrane without mRNA or protein synthesis. ${ }^{21-23}$

Bcl-2 and Bcl- $\mathrm{x}_{\mathrm{L}}$ inhibit the oligomerization of Bax and prevent apoptosis by preserving mitochondrial integrity and suppressing cytochrome $c$ release without ameliorating ATP depletion. ${ }^{18}$ As our data demonstrate, there were no differences in the level of $\mathrm{Bcl}-2$ in either group. In contrast, however, we have found higher levels of $\mathrm{Bcl}-\mathrm{x}_{\mathrm{L}}$ proteins in the newborn cardioplegic group compared with in their mature counterparts. This suggests that the newborn myocardium has increased levels of antiapoptotic factors (eg, Bcl- $\mathrm{x}_{\mathrm{L}}$ ) despite being in the proapoptotic state (higher Bax/ Bcl-2 ratio). Despite increased levels of Bcl- $\mathrm{x}_{\mathrm{L}}$, however, cytochrome $c$ is released within 6 hours from the mitochondria of the postcardioplegic neonatal lamb. 
Reactive oxygen species are critical signaling molecules in regulating growth, survival, and death pathways. In the cardiovascular system significant amounts of reactive oxygen species are generated as byproducts of mitochondrial metabolism and might increase to toxic levels in the myocardium during and after periods of ischemia. ${ }^{24}$ ASK-1 is a member of the mitogen-activated protein kinase kinase kinase family, which is activated in response to cytokines, oxidants, and stress signals. It is also an upstream activator of p38 mitogen-activated protein kinases and c-Jun NH2terminal kinase, contributing a pivotal signaling pathway in apoptosis. ${ }^{25,26}$ Under resting conditions, ASK-1 exists in the cytosol bound to a small redox-sensitive protein, thioredoxin. In the presence of oxidative stress, oxidation of thioredoxin results in dissociation from ASK-1, leaving ASK-1 in its active form. ${ }^{27}$ This study has demonstrated greater ASK-1 activity in the neonatal cardioplegic group, suggesting greater dissociation of thioredoxin from ASK-1 caused by the greater presence of oxygen free radicals or diminished ability to ameliorate free radical injury in the neonatal hearts. Oxidative stress might be an important initiating event in the induction of neonatal myocardial apoptosis. $^{28,29}$

These data support a profile of increased vulnerability of the neonate to apoptosis-related processes in the early postoperative period by using a clinically relevant model of cardioplegic arrest. The mitochondrial release of cytochrome $c$ suggests the possibility that, in addition to classical myocardial stunning, apoptosis-related mitochondrial dysfunction might contribute to the midnight sag frequently noted after neonatal cardiac surgery. Consequently, redesign of presently available myoprotective strategies might allow improved preservation of mitochondrial integrity in the postoperative neonatal heart.

\section{References}

1. Wernovsky G, Wypij D, Jonas RA, et al. Postoperative course and hemodynamic profile after the arterial switch operation in neonates and infants. A comparison of low-flow cardiopulmonary bypass and circulatory arrest. Circulation. 1995;92:2226-35.

2. Hoffman TM, Wernovsky G, Atz AM, et al. Efficacy and safety of milrinone in preventing low cardiac output syndrome in infants and children after corrective surgery for congenital heart disease. Сirculation. 2003;107:996-1002.

3. Abdelwahid E, Pelliniemi LJ, Niinikoski H, et al. Apoptosis in the pattern formation of the ventricular wall during mouse heart organogenesis. Anat Rec. 1999;256(2):208-17.

4. Bornicki RA, Backer CL, Baden HP, Mavroudis C, Crawford SE, Green TP. Dexamethasone reduces the inflammatory response to cardiopulmonary bypass in children. Ann Thorac Surg. 2000;69(5):1400-5.

5. Anversa P, Cheng W, Lin Y, Lori A, Redaelli G, Kajstura J. Apoptosis and myocardial infarction. Basic Res Cardiol. 1998;93(suppl 3):8-12.

6. Goldstein JC, Waterhouse NJ, Juin P, Evan GI, Green DR. The coordinate release of cytochrome $c$ during apoptosis is rapid, complete and kinetically invariant. Nat Cell Biol. 2000;2:156-62.

7. Ricci JE, Gottlieb RA, Green DR. Caspase-mediated loss of mitochondrial function and generation of reactive oxygen species during apoptosis. J Cell Biol. 2003;160(1):65-75.
8. Olivetti G, Quaini F, Sala R, et al. Acute myocardial infarction in humans is associated with activation of programmed myocyte cell death in the surviving portion of the heart. $\mathrm{J} \mathrm{Mol} \mathrm{Cell} \mathrm{Cardiol.}$ 1996;28:2005-16.

9. Ruetten H, Badorff C, Ihling C, et al. Inhibition of caspase-3 improves contractile recovery of stunned myocardium, independent of apoptosis-inhibitory effects. J Am Coll Cardiol. 2001;38:2063-70.

10. Schmitt JP, Schroder J, Schunkert H, Birnbaum DE, Aebert H. Role of apoptosis in myocardial stunning after open heart surgery. Ann Thorac Surg. 2002;73:1229-35.

11. Hammel JM, Caldarone CA, VanNatta TL, et al. Myocardial apoptosis after cardioplegic arrest in the neonatal lamb. $J$ Thorac Cardiovasc Surg. 2003;125:1268-75.

12. Casey WF, Hauser GJ, Hannallah RS, et al. Circulating endotoxin and tumor necrosis factor during pediatric cardiac surgery. Crit Care Med. 1992;20:1090-6.

13. Cryns V, Yuan J. Proteases to die for. Genes Dev. 1998;12:1551-70.

14. Kroemer G, Reed JC. Mitochondria control of cell death. Nat Med. 2000;6:513-9.

15. Vaux DL, Strasser A. The molecular biology of apoptosis. Proc Natl Acad Sci U S A. 1996;93:2239-44.

16. Tsujimoto Y. Role of Bcl-2 family proteins in apoptosis: apoptosomes or mitochondria? Genes Cell. 1998;3:697-707.

17. Martinou JC, Green DR. Breaking the mitochondrial barrier. Nat Rev Mol Cell Biol. 2001;2:63-6.

18. Bishorpric NH, Andreka P, Slepak T, Webster KA. Molecular mechanism of apoptosis in the cardiac myocyte. Curr Opin Pharmacol. 2001;1:141-50.

19. Narula J, Pandey P, Arbustini E, et al. Apoptosis in heart failure: release of cytochrome $c$ from mitochondria and activation of caspase-3 in human cardiomyopathy. Proc Natl Acad Sci U S A. 1999;96:81448149.

20. Ricci JE, Gottlieb RA, Green DR. Caspase-mediated loss of mitochondrial function and generation of reactive oxygen species during apoptosis. J Cell Biol. 2003;160:65-75.

21. Narita M, Shimizu S, Ito T, et al. Bax interacts with the permeability transition pore to induce permeability transition and cytochrome $c$ release in isolated mitochondria. Proc Natl Acad Sci U S A. 1998;95: 14681-6.

22. Liu X, Kim CN, Yang J, Jemmerson R, Wang X. Induction of apoptotic program in cell-free extracts: requirement for dATP and cytochrome c. Cell. 1996;86:147-57.

23. Sakahira H, Enari M, Nagata s. Cleavage of CAD inhibitor in CAD activation and DNA degradation during apoptosis. Nature. 1998;391: 96-9.

24. Matsuzawa A, Ichijo H. Molecular mechanisms of the decision between life and death: regulation of apoptosis by apoptosis signalregulating kinase 1. J Biochem. 2001;130(1):1-8.

25. Ichijo H, Nishida E, Irie K, et al. Induction of apoptosis by ASK1, a mammalian MAPKKK that activates SAPK/JNK and p38 signaling pathways. Science. 1997;275(5296):90-4.

26. Tobiume K, Matsuzawa A, Takahashi T, et al. ASK1 is required for sustained activations of JNK/p38 MAP kinases and apoptosis. EMBO Rep. 2001;2(3):222-8.

27. Liu Y, Min W. Thioredoxin promotes ASK-1 ubiquitination and degradation to inhibit ASK1-mediated apoptosis in a redox activityindependent manner. Circ Res. 2002;90:1259-66.

28. Hatai T, Matsuzawa A, Inoshita S, et al. Execution of apoptosis signal-regulating kinase 1 (ASK1)-induced apoptosis by the mitochondria-dependent caspase activation. J Biol Chem. 2000;275(34):2657681.

29. Cook SA, Sugden PH, Clerk A. Regulation of Bcl-2 family proteins during development and in response to oxidative stress in cardiac myocytes: association with changes in mitochondrial membrane potential. Circ Res. 1999;85:940-9.

\section{Discussion}

Dr Ralph J. Damiano, Jr (St Louis, Mo). You have beautifully shown that in the newborn hearts you had more apoptosis, but it is possible that they are maybe just more apoptotically active. What 
data do you have to say that they are more vulnerable to apoptosis than a mature model? Do you follow me? You could have apoptosis, but if it was tolerated physiologically, I think that is an important differentiation. And how would you answer that, and what data do you have to suggest that it is actually more vulnerable to apoptosis?

Dr Mohsen Karimi (Iowa City, Iowa). That is an excellent question. We know that neonates are vulnerable to apoptosis because it is part of their normal development. We have demonstrated previously that they became more proapoptotic after cardioplegic arrest when you even compare them with neonates undergoing only cardiopulmonary bypass without cardioplegic arrest.

Our final discussion slide also proves the above point that neonatal lambs underwent more apoptosis as the result of cardioplegic arrest even when compared with the mature operated lambs and nonoperated groups. As you see on this slide, the concentrations of cytosolic cytochrome $c$ were the same for both the mature cardioplegic and mature nonoperated groups. But when you compare the newborn cardioplegic versus newborn nonoperated groups, there was significantly higher cytochrome $c$ release in the newborns that underwent cardioplegic arrest. This basically demonstrates that neonatal cardioplegic groups are undergoing more apoptosis than the nonoperated neonate.

Dr Frank W. Sellke (Boston, Mass). Is all of the cytosolic cytochrome released from apoptosis, or is there a component to necrosis causing this?

Dr Karimi. We think it is from apoptosis. We have hematoxylin and eosin staining from our sections that did not demonstrate any necrotic histology.

Dr Sellke. Your first statement in your conclusion was that the $\mathrm{Bax} / \mathrm{Bcl}-2$ ratio is the cause for this differential effect between the mature and the immature hearts, yet the slide that demonstrated the comparison between the neonatal and the mature heart before and after cardioplegia had a similar trend in the mature and immature heart. If you had increased the numbers, I suspect that both of these would be statistically significant.
Dr Karimi. That is a true statement. The presence of a higher $\mathrm{Bax} / \mathrm{Bcl}-2$ ratio in neonates might or might not contribute to this process of apoptosis, but there are other proapoptotic factors, such as Bid, truncated Bid, and Bad, which we are actively investigating.

Dr Antonio F. Corno (Lausanne, Switzerland). When we study the effects of ischemia and reperfusion on the myocardium, I am sure you are aware that we should evaluate all the components not only during ischemia, including the type of myocardial protection, duration of cardioplegia, type of components, cardioplegia pressure and volume infusion, and so forth, but even during the reperfusion, the controlled versus noncontrolled reperfusion.

How did you consider all these variables in your protocol? What is the rationale to study the status of myocardium after a period of 6 hours of recovery instead of at the end of reperfusion?

Dr Karimi. I am sorry, but there was a lot of echo. I could not get the first question, sir.

Dr Corno. How did you evaluate all the variables during ischemia and during reperfusion in terms of type of myocardial protection and type of reperfusion in your protocol?

Dr Karimi. If I understand the question well, you are asking how we differentiate between myocardial ischemia before reperfusion or after reperfusion. Is that what you are asking?

Dr Corno. You compared the neonatal hearts with the mature myocardium, and we know that there is a huge difference between the two. You used the same type of myocardial protection for both, knowing that there is a difference. Do you think this is important in the results you have seen?

Dr Karimi. Yes, it is important.

Dr Corno. Second question. Why did you decide to study the myocardium after a period of 6 hours of recovery instead of studying the effects at the end of reperfusion? Why did you leave a 6-hour recovery?

Dr Karimi. We tried to do 6 hours to simulate what is out in the real clinical scenario. The neonatal heart function usually deteriorates after 6 to 12 hours from the operation, and we tried to create the same clinical scenario. 